\title{
Digital human factor management
}

\author{
Yuri Ivanov ${ }^{1}$, Alexei Voroshilov ${ }^{2 *}$, Nikolai Novikov ${ }^{3}$, and Konstantin Todradze ${ }^{3}$ \\ ${ }^{1}$ SUEK-Kuzbass, 1, Vasilieva, Leninsk-Kuznetskiy, Russia \\ ${ }^{2}$ Kuzbass-COT, 1, Sosnoviy Bulvar, Kemerovo, Russia \\ ${ }^{3}$ NACOT, 29, 4-ya Parkovay, Moscow, Russia
}

\begin{abstract}
Now culture of manage safety, health and wellbeing in the mining industry needs a scientifically sound new approach to the training process for professionals at all levels. Insufficient qualification of employees and managers in occupational safety, health and wellbeing has a negative impact on the occupational injury rate, and the current occupational safety, health and wellbeing management system does not allow to instill in employees compliance with the requirements for their own safety. To improve the quality of employees training in safe work practices and to ensure their health, safety and wellbeing in processes, a new training process has been developed and implemented based on a video-based continuous learning system. The innovation will make it possible to manage the human factor by improving employees competence, increasing the efficiency of the system OSH management, taking prompt corrective action, reducing injury rates and retaining human resources.
\end{abstract}

\section{Introduction}

Despite the general tendency towards a decrease in the number of accidents, the risk of underground coal mining injury in the coal mining industry remains among the highest both in Russia and abroad. Moreover, numerous researches [1-3] show specifically the human factor to cause $70-90 \%$ of incidents, disasters, and accidents.

Instigating a strong safety culture can serve as an important instrument in reducing the number of workplace incidents, injuries, and disasters.

The notion of a safety culture first appeared in IAEA documentation after the causes of the Chernobyl disaster had been analyzed [4]. Subsequently, ILO clarified and expanded upon it in its Convention No. 187, making it suitable for all countries, industries, companies, employers and employees:

(d) the term a national preventative safety and health culture refers to a culture in which the right to a safe and healthy working environment is respected at all levels, where government, employers and workers actively participate in securing a safe and healthy working environment through a system of defined rights, responsibilities and duties, and where the principle of prevention is accorded the highest priority. [5]

\footnotetext{
* Corresponding author: besimply@yandex.ru
} 
The authors think that in case of technical systems, prevention is technical maintenance, and as for the employee - it is first and foremost training in safe work practices.

In its documents [6] the IAEA has defined the following stages of safety culture development and improvement:

(1) Safety is compliance driven and is based mainly on rules and regulations. At this stage, safety is seen as a technical issue, whereby compliance with externally imposed rules and regulations is considered adequate for safety.

(2) Good safety performance becomes an organizational goal and is dealt with primarily in terms of safety targets or goals.

(3) Safety is seen as a continuing process of improvement to which everyone can contribute.

Thus, while the first two stages deal with technicalities and organizational issues, the third one mainly focuses on each employee, and on the human factor to that end.

As there exists a substantial amount of definitions of the Human Factor, we will provide the one we will be using in this work [7].

Human Factor is the difference between the Actual Set of Abilities and the Required Set of Abilities that changes over time.

Actual Set of Abilities is a demonstrable ability to safely perform specific work, which is based on the intellectual, sensory, protective, and physical principal components.

Required Set of Abilities is the entirety of requirements to the intellectual, sensory, protective, and physical human abilities necessary to perform specific work.

This definition can be reduced to a simple equation:

$$
H F=A S A-R S A
$$

where:

HF - Human Factor;

RSA - Required Set of Abilities;

ASA - Actual Set of Abilities.

A switch from the qualitative assessments of the Human Factor to the quantitative ones requires quantification of the elements the Human Factor comprises, that is expressing qualitative traits parameters through numeric values.

Then assessment of the Human Factor through the formula (1) produces very interesting results:

- if ASA $<$ RSA, then HF is negative, in which case the value of the Human Factor is negative and it is categorized a danger and an occupational hazard;

- if ASA=RSA, then HF is neutral, the value of the Human Factor is neutral, and the probability of an accident is within the acceptable levels;

- if ASA $>$ RSA, then HF is positive, and, as a rule, the actions of the employee are supposed to ensure quality and the utilization of safe work practices.

Thus, within the proposed approach, the value of the Human Factor can be not only negative, as under traditional understandings of the Human Factor, but also neutral or positive.

Assuming that the terms "Set of Intellectual Abilities" and "Competence" refer to one and the same - the ability of an employee to perform their duties in compliance with the health and safety regulations, let us switch to the more widespread notion - the competence of an employee.

The digital technologies of continuous training are the most promising when it comes to maintaining the competence of an employee at a level necessary to utilize safe work practices. Note that the term "digital technologies of training" is a specific instance of information 
technology, and the term "digital technologies of training" accentuates that the training process happens through computers.

\section{Research}

Technology of managing the Human Factor digitally through continuous accelerated training of employees in safe work practices has been developed on the basis of the safety culture principles and the new Human Factor concept [7].

The first time Kuzbass-COT enacted this approach was at SUEK-Kuzbass facilities through implementing the "Video and Information Complex of Continuous Development and Control of the Employee Competence in the Field of Occupational Safety, Health and Wellbeing" (hereinafter the Complex) [8,9].

The Complex mainly serves to significantly reduce workplace injuries through management of the Human Factor - management of the employee competence.

At the core of the Complex is the Methodology of mass development and control of the employee competence, as per OSH requirements, developed by Kuzbass-COT, using the latest innovations in training of adults. This Methodology ensures the required level of employee competence in the field of occupational health, safety and wellbeing, through the use of innovation technologies of custom individual employee training courses. Those technologies account for the psychophysiological specifics of absorption and retention of information by humans, and guarantee efficient mastering of the material. The Methodology allows to plant the stereotypes (skills) of safe work practices, correct emergency actions, and skills of competent first aid into the consciousness of employees on a mass scale.

The specific feature of this Methodology is in that it enables soft competence enforcement - with no reprimands, fines, or other disciplinary actions.

Let us give a short description of an accelerated training session.

Before the shift the employee is given, in accordance with their occupation, a problem on a computerized terminal (Figure 1), which they are to solve by selecting one of the three suggested answers.

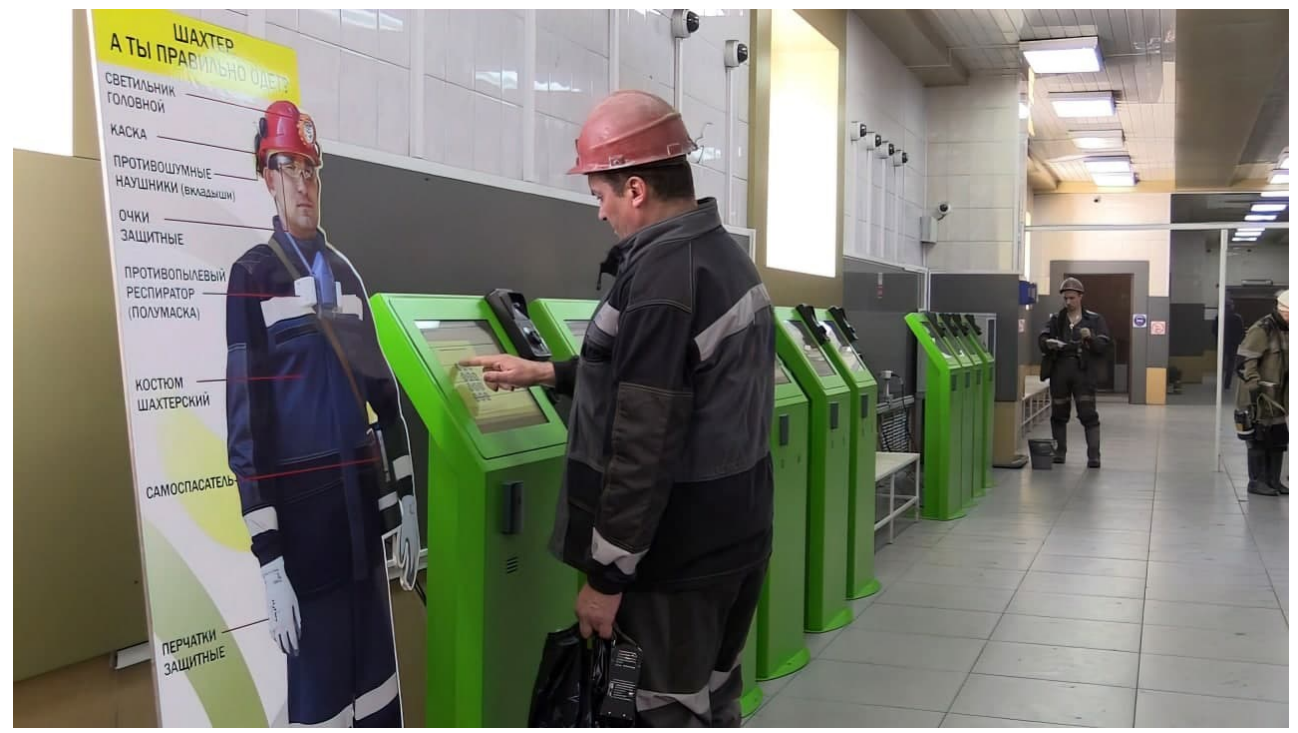

Fig. 1. Employees undergoing accelerated training at one of the SUEK-Kuzbass coal mines before their shifts 
If they solve the problem incorrectly, the employee is shown a video of possible development of the hazardous situation, as well as repercussions - damage of various internal organs the employee may sustain as a result of their wrongful actions. Next, the correct answer is highlighted to the employee to adjust their competence. Next, the employee is given the problem they failed to solve again. Once it has been solved correctly, the employee is given hidden praise through the demonstration of injuries they have avoided by solving the problem correctly.

To assess the degree of the required competence and to asses the Human Factor, use the criteria listed in Table 1.

The required "competent" competence level is "over $90 \%$ of the OSH problems solved correctly."

The actual competence level of an employee is determined through a fraction, represented as the percentage of problems solved correctly.

For each employee the Human Factor is assessed through the formula:

where:

$$
\mathrm{HF}_{\mathrm{I}}=\mathrm{ASA}_{\mathrm{I}}-\mathrm{RSA}_{\mathrm{I}}
$$

$\mathrm{HF}_{\mathrm{I}}$ - Human Factor;

$\mathrm{RSA}_{\mathrm{I}}$ - Required Competence Level;

$\mathrm{ASA}_{\mathrm{I}}-$ Actual Competence Level.

For an easy Human Factor management, the Complex uses three competence levels: competent, poorly competent, and incompetent. See Table 1 for $\mathrm{HF}_{\mathrm{I}}$ assessment.

Table 1. Human Factor assessment criteria in accordance with competence levels

\begin{tabular}{|l|c|c|c|}
\hline $\begin{array}{c}\text { Competence } \\
\text { Level }\end{array}$ & $\begin{array}{c}\text { Actual Competence, } \\
\mathrm{ASA}_{\mathrm{I}}\end{array}$ & $\begin{array}{c}\text { Required } \\
\text { Competence, } \\
\mathrm{RSA}_{\mathrm{I}}\end{array}$ & Human Factor \\
\hline competent & $\mathrm{ASA}_{\mathrm{I}} \geqslant 90$ & $\mathrm{RSA}_{\mathrm{I}}=90$ & $\mathrm{HF}_{\mathrm{I}} \geqslant 0$ \\
\hline poorly competent & $80 \leqslant \mathrm{ASA}_{\mathrm{I}}<90$ & $\mathrm{RSA}_{\mathrm{I}}=90$ & $\mathrm{HF}_{\mathrm{I}}<0$ \\
\hline incompetent & $<80$ & $\mathrm{RSA}_{\mathrm{I}}=90$ & $\mathrm{HF}_{\mathrm{I}} \ll 0$ \\
\hline
\end{tabular}

A study of the distribution of accident victims among the employees into competence groups over the 2016-2018 period (among those who had taken the accelerated tests before their shifts) found a connection between the number of accidents and the competence of employees in the OSH field, as well as demonstrated that:

- the poorly competent employees are 2 times more likely to be injured than the competent employees;

- the incompetent employees are 6.7 times more likely to be injured than the competent employees.

This data confirms that the competence level criteria are justified.

Where the results of the accelerated training indicate that the value of the Human Factor is negative, the Complex automatically schedules the employee for additional accelerated training:

- the employees with poor results are automatically scheduled on a monthly basis for an accelerated training test, consisting of five problems;

- violators of labor ethics are automatically scheduled for solving five problems related to accidents analysis or a shocking video. 
Using the connection between the unsafe actions by employees and the number of accidents, Kuzbass-COT has developed a methodology for numeric evaluation of the occupation injury risks for employees, caused by their personal incompetent actions. This methodology is used at the facilities where the Complex has been installed to predict the number of injured employees in course of a year.

The Complex sends to the employees and to the supervisors of all levels information, characterizing the state of competence, as well as the professional risks for SUEK-Kuzbass, JSC production units, sites, employees.

The generated employee-level record includes the following information:

- history of competence dynamics for the year;

- notes and recommendations for the competence level of each employee;

- how many times the employee risk exceeded the target risk in the previous month;

- competence value in the previous month.

The following information is forwarded monthly through the web interface of the Complex to the head of the site:

- summary of the average competence level among the employees in the form of a table for the site on the whole;

- lists of employees automatically scheduled for an additional accelerated training test;

- personal competence level data for employees;

- list of employees with a persistent low competence level;

- predicted number of injured employees by the end of the year;

- information submitted by employees on instances of OSH violations (feedback).

The following information is forwarded monthly through the web interface of the Complex to the head of the facility:

- summary of the average competence level among the employees in the form of charts for the facility on the whole;

- summary of the average competence level among the employees for each site and the facility on the whole;

- predicted number of injured employees by the end of the year;

- summary of the numbers of employees automatically scheduled for an additional accelerated training, for each site and the facility.

For the purposes of competence dynamics analysis and control, the employees of the head company administration, industrial safety directorate, personnel management has an access to the web interface of the Complex in the "Employees Risks and Competence" section, where the following information is displayed:

- summary of the average employee competence level for each facility and the company on the whole;

- summary of the average employee competence level for each site and the facility on the whole;

- summary of the number of employees automatically scheduled for an additional accelerated training, "Extraordinary Video Instructions" and notes pertaining to their completion for each site and the facility on the whole;

- information submitted by employees on instances of OSH violations and measures taken by the heads of the production units to rectify them;

- information on the average competence of employees for each production unit and the company on the whole;

- fatal injury modifiers, injury frequencies;

- before-the-shift test results data, for each production unit;

- predicted number of injured employees by the end of the year for each facility and the company on the whole; 
- information submitted by employees on instances of OSH violations and measures taken by the heads of the sites to rectify them.

Thus, the digital technologies of continuous training of employees allow to instill safe industrial work practices into employees on a mass scale.

Let us look at the results of the Complex being put into practice for managing the Human Factor at SUEK-Kuzbass, JSC facilities, where employees have undergone training over three million times.

Figure 2 shows the dynamics of change in the number of accidents at SUEK-Kuzbass, JSC for each year. This includes both those facilities where the Complex was launched at the beginning of 2015 and the facilities where the Complex is not installed.

As the competence of employees grew, the number of accidents at the facilities with the Complex installed rapidly declined. By 2019 the number of accidents had decreased 3 times. Meanwhile, the data on the number of accidents at the other SUEK-Kuzbass, JSC facilities where the Complex had not been installed shows no positive dynamics in the number of accidents. This proves the Complex to have independently and significantly lowered the number of accidents.

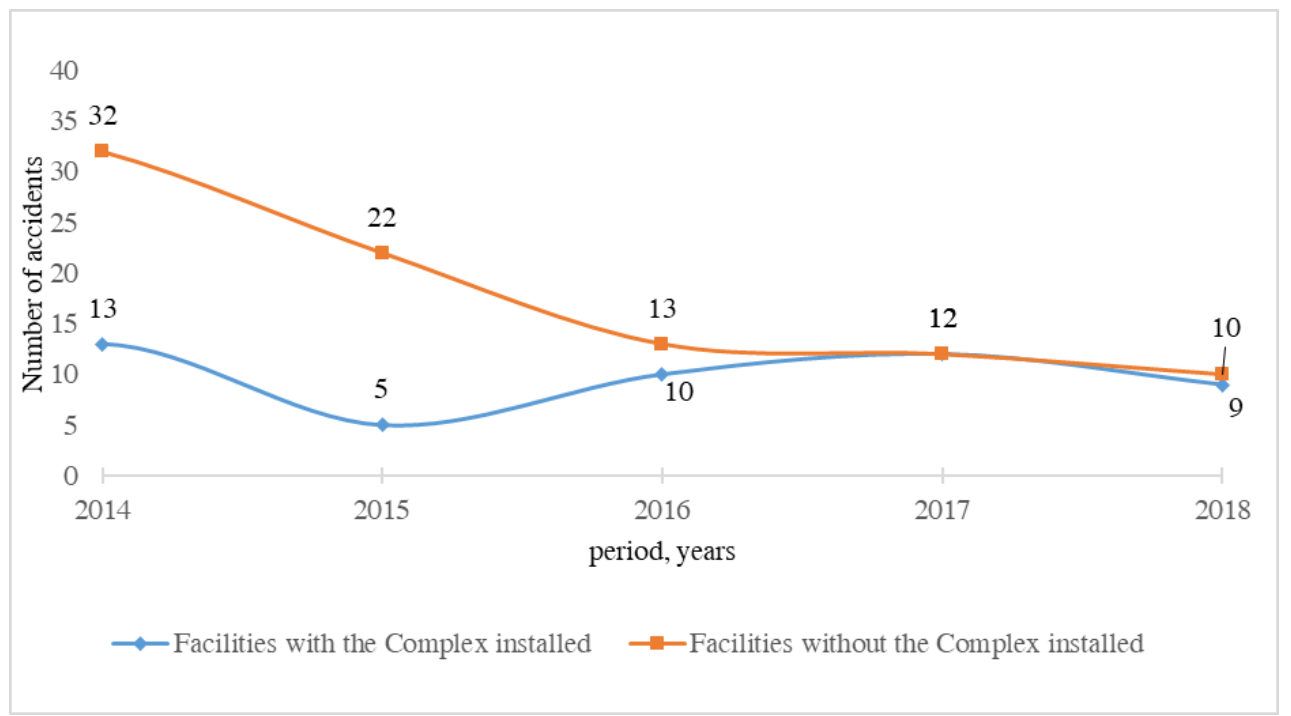

Fig. 2. Dynamics in the number of accidents after the implementation of the Complex in 2015

Presently, 10-12 thousand employees are undergoing daily accelerated trainings at 50 various facilities owned by 15 different large and medium companies. On the whole, around 15 million accelerated trainings have taken place. There is an observable steady 1.5-3 time decline in the number of accidents over 2-3 years.

ISSA mining experts have concluded the Kuzbass-COT technology to meet the criteria of "The Prevention of Workplace Accidents. Zero Vision in the Field of Injury" Programs, and given to the Kuzbass-COT Methodology an "ISSA Mining Excellence" quality award.

\section{Conclusion}

A new approach to the digital human factor management has been proposed, whose essence is continuous accelerated training of employees in matters of $\mathrm{OSH}$, using the video and information computer system. 
Principal procedures of the continuous accelerated training of employees in matters of OSH have been described.

Data, showing the continuous accelerated training of employees in the occupational health and safety to be highly efficient, and to decrease the number of accidents 1.5-3 times over the course of 2-3 years has been supplied.

\section{References}

1. A. Filho, The Impacts of Human Factors in Fatal Workplace Accidents. (2012)

2. S. Salminen, Accidents: Causes, Analysis and Prevention. 87-103. (2009)

3. B. Fabiano, M. Pettinato, A. Reverberi, F. Currò, Chemical Engineering Transactions, 77, 283-288 (2019).

4. Summary Report on the Post-Accident Review Meeting on the Chernobyl Accident. Safety Series, No. 75-INSAG-1 (Vienna, 1986)

5. C187 - Promotional Framework for Occupational Safety and Health Convention, ILO (2006)

6. Key practical issues in strengthening safety culture: INSAG-15 / a report by the International Nuclear Safety Advisory Group (Vienna, 2002)

7. Y. Voroshilov. Scientific substantiation and development of technical solutions for monitoring the dust situation of coal mines included the human factor. (Kemerovo, Russia, 2020)

8. Y. Ivanov, L. Un, A. Voroshilov, G. Sedelnikov, S. Voroshilov, Occupational Safety in Industry, 2, 79-83, (2017)

9. V. Lisovskiy, Y. Ivanov, A. Voroshilov, G. Sedelnikov, UGOL', 12, 41-46, (2018) 